\title{
Avances en inmunoterapia intralinfática, enfermedad respiratoria exacerbada por aspirina y prevención en enfermedades alérgicas
}

\author{
Advances in intralymphatic immunotherapy, \\ aspirin-exacerbated respiratory disease \\ and prevention of allergic disease
}

\author{
Dra. Rosa Elena Huerta Hernández* \\ *Alergólogo Pediatra. Clínica de Alergia Pediátrica. Pachuca, Hidalgo.
}

\begin{abstract}
Citar como: Huerta HRE. Avances en inmunoterapia intralinfática, enfermedad respiratoria exacerbada por aspirina y prevención
\end{abstract} en enfermedades alérgicas. Alergia Asma Inmunol Pediatr. 2021; 30 (1): 3-4. https://dx.doi.org/10.35366/100110

En las publicaciones de este número, veremos temas de importancia para la práctica clínica en pediatría, alergia e inmunología.

En el artículo de revisión La enfermedad respiratoria exacerbada por aspirina y la desregulación de los mastocitos, los autores mencionan el tratamiento integral de la enfermedad y el uso con terapia biológica; así mismo, hacen énfasis en la dificultad para realizar el diagnóstico en la población pediátrica. La enfermedad respiratoria exacerbada por la aspirina debe considerarse y evaluarse en pacientes con asma pediátrica con pólipos nasales y/o intolerancia a los inhibidores de la COX-1. Se puede requerir un reto con aspirina para confirmar el diagnóstico y poder ofrecer el tratamiento específico disponible para la población pediátrica. ${ }^{1}$

En Neutropenia congénita, se realiza una amplia revisión sobre los trastornos congénitos asociados con neutropenia, los que pueden ocurrir de forma aislada o como característica de los trastornos inmunes más complejos y específicamente en los defectos cuantitativos de los neutrófilos. Algunos de estos trastornos son neutropenia étnica benigna, mutaciones autosómicas dominantes del gen ELANE —el cual codifica la elastasa de los neutrófilos-, deficiencia GRI1, síndrome de Barth, entre otros.

La neutropenia se considera como una afección peligrosa y potencialmente mortal que hace que los pacientes sean vulnerables a infecciones recurrentes. Su gravedad es proporcional al recuento absoluto de granulocitos neutrófilos en la circulación. En pacientes pediátricos, la neutropenia puede tener muchas etiologías diferentes. En las últimas décadas se ha descubierto una serie de genes que son responsables de la neutropenia congénita. Mediante la perturbación del metabolismo de la energía mitocondrial, el tráfico de vesículas o la síntesis de proteínas funcionales, estas mutaciones causan un arresto de maduración en las células precursoras mieloides en la médula ósea. Aparte de estas formas aisladas, la neutropenia congénita se asocia con una multiplicidad de síndromes que incluye, entre otras, albinismo oculocutáneo, enfermedades metabólicas y síndromes de insuficiencia de la médula ósea. La neutropenia congénita es una inmunodeficiencia primaria que se asocia con infecciones bacterianas recurrentes, fenómenos autoinflamatorios y

\footnotetext{
Correspondencia:

Dra. Rosa Elena Huerta Hernández Alergólogo Pediatra

Clínica de Alergia Pediátrica. Pachuca, Hidalgo.

Artículo 27 Núm. 102, Col. Fraccionamiento Constitución, 42080, Pachuca de Soto, Hidalgo, México. E-mail: rehhmexico@gmail.com
} 
autoinmunes, neoplasias malignas hematológicas y manifestaciones neuropsiquiátricas. ${ }^{2}$

En Factores de riesgo socioambientales asociados a la presencia de síntomas de atopias en niños menores de tres años, los autores encontraron en su estudio que los factores socioambientales asociados al riesgo de desarrollo de atopias fueron: tener un familiar alérgico, uso de terapia antibiótica en el primer año de vida, así como la temporalidad lluviosa en Quito, Ecuador, para asma y rinitis alérgica, y la estación seca para dermatitis atópica. En una revisión sistemática sobre los factores de riesgo para asma y rinitis alérgica en población pediátrica, se encontró una correlación estadísticamente significativa entre los niños con rinitis alérgica y asma, y la exposición ambiental a hidrocarburos aromáticos policíclicos, quienes viven en ciudades industrializadas con tráfico elevado, humedad y exposición a humedad, género masculino, polimorfismos de nucleótidos únicos en los genes PTNP22 y CTLA-4, consumo de alimentos procesados y productos de margarina, uso de paracetamol en el último año, historia de tuberculosis, atopia parental, uso de antibióticos e infecciones in utero, antecedentes de alimentación de fórmulas infantiles lácteas y cesárea. Se tuvo en cuenta un vínculo fuerte y complejo entre rinitis alérgica y asma. El asma representa un factor de riesgo importante para la aparición de rinitis alérgica, que se correlaciona con síntomas asmáticos más graves; incluso la aparición de asma en un niño con rinitis alérgica lo empeora. Se encontró que la interacción de factores de riesgo genéticos y ambientales, así como los consiguientes cambios epigenéticos, microbiotas e inmunológicos, condujeron al desarrollo de rinitis y asma en niños con vías atópicas y no atópicas. Un seguimiento estrecho de los factores de riesgo evidenciados puede ayudar con un diagnóstico y tratamiento tempranos en pacientes con rinitis alérgica. ${ }^{3}$

En la revisión Inmunoterapia intralinfática para el manejo de patologías alérgicas en el paciente pediátrico. Revisión sistemática de ensayos clínicos, se considera que la inmunoterapia intralinfática (IIL) es una estrategia terapéutica efectiva para el tratamiento de enfermedades alérgicas en pacientes pediátricos y adultos, que induce mejorías en las pruebas de provocación nasal, disminuye los síntomas locales y sistémicos, en la mayoría de los casos conduce a la reducción del consumo de medicamentos, mejora parámetros inmunológicos y puede mejorar la calidad de vida. Además, se asocia con buena adherencia terapéutica y eventos adversos leves, pero no moderados ni severos. Sin embargo, los autores comentan que falta realizar estudios para evaluar la efectividad de la IIL en otras enfermedades alérgicas, y elaborar estudios específicos en pacientes de 15 años o menos, así como determinar cada cuánto tiempo es necesario realizar otras aplicaciones de la IIL, ya que los estudios realizados hasta ahora sólo han dado seguimiento máximo de un año.

En el trabajo publicado por Weinfeld y colaboradores, ${ }^{4}$ se sugiere que el uso de un refuerzo prestacional, junto con tres inyecciones iniciales de IIL, podría ser de utilidad para mantener elevados los niveles de lgG4 específicos, junto con disminución en los síntomas nasales y oculares en pacientes con rinoconjuntivitis alérgica.

Los autores de este artículo sugieren que se podrían esperar más beneficios de los refuerzos preestacionales en términos de una mayor duración de la eficacia del tratamiento; no obstante, el concepto de un refuerzo estacional necesita ser desarrollado aún más, antes de que pueda ser introducido en la práctica clínica.

Puede ser más fácil, a pesar de la considerable heterogeneidad, establecer un algoritmo clínico basado en la respuesta específica de dosis alta de IgG4 a la IIL que para la inmunoterapia subcutánea específica. El algoritmo puede incluir una combinación de aumento absoluto y relativo en IgG4 específico. Sobre la base de los niveles específicos de IgG4 que fluctúan en relación con la IIL, también proponen la monitorización de lgG4, la cual puede llegar a convertirse en una herramienta futura que se puede utilizar para decidir el número de inyecciones de refuerzo que un paciente individual podría necesitar para experimentar un efecto clínico suficiente y mantenido. ${ }^{4}$

\section{REFERENCIAS}

1. Tuttle KL, Schneider TR, Henrickson SE, Morris D, Abonia JP, Spergel JM et al. Aspirin-exacerbated respiratory disease: not always "adult- onset". J Allergy Clin Immunol Pract. 2016; 4 (4): 756-758.

2. Spoor J, Farajifard H, Rezaei. Congenital neutropenia and primary immunodeficiency diseases. Crit Rev Oncol Hematol. 2019; 133. 149-162.

3. Testa D, Di Bari M, Nunziata M, De Cristofaro F, Massaro G, Marcuccio G, Motta G. Allergic rhinitis and asthma assessment of risk factors in pediatric patients: A systematic review. Int J Pediatr Otorhinolaryngol. 2020; 129: 109759.

4. Weinfeld D, Westin U, Hellkvist L, Mellqvist UH, Jacobsson I, Lars $\square$ Olaf Cardell LO. A preseason booster prolongs the increase of allergen specific IgG4 levels, after basic allergen intralymphatic immunotherapy, against grass pollen seasonal allergy. Allergy Asthma Clin Immunol. 2020; 16: 31. Available in: https://doi.org/10.1186/ s13223-020-00427-z. 